\title{
Facile Rapid Synthesis of Polyaniline (PANI) Nanofibers
}

\author{
Ashok D. Bhagwat ${ }^{1,2}$, Sachin S. Sawant ${ }^{1,3}$, Chandrashekhar M. Mahajan ${ }^{3}$ \\ 1 Singhania University, Pacheri Bari, Jhunjhunu-333515 Rajasthan, India \\ ${ }_{2}^{2}$ Dnyanshree Institute of Engineering and Technology, Satara - 415013 Maharashtra, India \\ ${ }^{3}$ Department of Engineering Sciences and Humanities, Vishwakarma Institute of Technology, Pune-411037 \\ Maharashtra, India
}

(Received 09 July 2015; revised manuscript received 09 March 2016; published online 15 March 2016)

\begin{abstract}
A Polyaniline (PANI) nanofibers have been successfully synthesized by a facile rapid oxidative polymerization of aniline hydrochloride and ammonium persulfate at high temperature $\left(60^{\circ} \mathrm{C}\right)$. The structural and optical properties of PANI nanofibers are investigated by using X-ray diffraction (XRD), UV-VIS Spectroscopy and Fourier Transform Infrared Spectroscopy (FTIR). The XRD analysis ascertains formation of PANI with nanocrystalline nature with average crystallite size $30 \mathrm{~nm}$. Further, FTIR pattern confirmed the formation of PANI. SEM analysis has revealed homogeneous fibrous morphology of PANI nanofibers, a well formed mesh of interconnected and entangled PANI nano-fibers over the scanned area. The UV-VIS spectroscopic analysis shows three major absorption peaks at 256.73, 361.17 and $480.95 \mathrm{~nm}$ confirmed the PANI formation with conducting state.
\end{abstract}

Keywords: Mesh, Nano-fibers, Polyaniline, Rapid mixing, Synthesis

DOI: $10.21272 /$ jnep.8(1).01037

PACS numbers: 68.37.Hk, 68.47.Mn, 82.35.Cd,

\section{INTRODUCTION}

Polyaniline (PANI) is the oldest and potentially one of the most useful conducting polymers because of its facile synthesis, environmental stability and simple acid/base doping/de-doping chemistry. Besides, low cost, high conductivity, and high pseudo-capacitance $[1,2]$ underlines its importance. It must be noted that nano-form of this polymer could offer new properties or enhanced performance hence nanostructured polyaniline (PANI) has attracted a great deal of interest during the past few years. The polyaniline nanostructures could be formed using simple method of chemical oxidative polymerization of aniline. Taking advantage of the unique reduction/oxidation chemistry of polyaniline and polyaniline/metal nanoparticle composites (nano-composites) could be of great use for various applications in ultrafast nonvolatile memory devices and for chemical catalysis. In addition, the use of polyaniline nanofibers or their composites can significantly enhance the sensitivity, selectivity, and response time of polyaniline-based chemical sensors. Recently conducting polymers found place as a gas sensor because of chief merits such as easy synthesis and room temperature operation Furthermore, an extensive range of potential technological applications of PANI includes storage batteries, electrochemical devices, light emitting diode, corrosion inhibitor and various types of chemical sensor and bio-sensors [2,3]. The first step towards realizing the applications of nano PANI and its composites is to synthesize the nano PANI films. The focus of this work is to study the standard method reported in literature (Kener et. al. [1]) with little or more variation in synthesis parameters to synthesize the nano-sized PANI powders and thin films.

\section{EXPERIMENTAL}

\subsection{Materials}

AR grade chemicals were used for synthesis of Poly- aniline (PANI) nano-fibers. Aniline Hydrochloride $\left(\mathrm{C}_{6} \mathrm{H}_{8} \mathrm{CIN}\right)$, Ammonium Persulfate $\left(\mathrm{NH}_{4}\right)_{2} \mathrm{~S}_{2} \mathrm{O}_{8}$ were purchased from Loba Chemie Pvt. Ltd. Mumbai, Maharashtra, India while Hydrochloric Acid ( $\mathrm{HCl}, 35-38 \%)$ was purchased from Thomas Baker Chemicals Pvt. Ltd. Mumbai, Maharashtra, India. All the reagents used in above mentioned methods used without any further purification for PANI synthesis.

\subsection{Method}

Typically, $1 \mathrm{~g}$ of aniline hydrochloride and $0.230 \mathrm{~g}$ of ammonium per-sulfate were dissolved in $20 \mathrm{ml}$ of double distilled water in two separate beakers. Both the solutions were heated up to $60{ }^{\circ} \mathrm{C}$ then rapidly mixed together to undergo polymerization. The reaction temperature was maintained at $60^{\circ} \mathrm{C}$ precisely for 5 minutes till the proper completion of polymerization process. This results into the formation of dark green colored emeraldin salt of polyaniline. Afterwards, heating was stopped and beaker containing PANI solution was allowed to cool naturally. No stirring and shaking was done throughout the synthesis. Later it was filtered and washed with distilled water and $0.2 \mathrm{M} \mathrm{HCl}$ to remove the impurities. Then a part of it was dried to get dark green emeraldin salt form of nanocrystalline PANI powder. The other part of PANI solution was then poured carefully drop wise (4-5 drops) on the cleaned and dried substrates and spread uniformly to form thin layer. All the substrates were first thoroughly washed with soap solution and rinsed with water. Then they were ultrasonicated in distilled water for $5 \mathrm{~min}$ and subsequently in $0.6 \mathrm{M} \mathrm{HCl}$ for $5 \mathrm{~min}$; and later washed by ethanol followed by distilled water. Subsequently substrates with PANI layer were dried at $52{ }^{\circ} \mathrm{C}$ temperature under IR illumination to obtain a uniform PANI film. Fig. 1 depicts photographic illustration of different steps involved in the synthesis of PANI nano-fibers. 


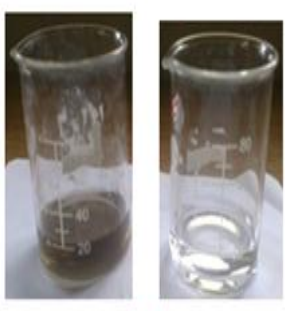

(a)

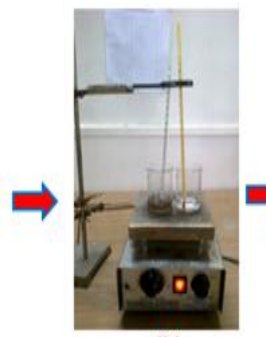

(b)

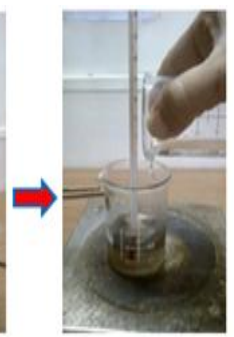

(c)

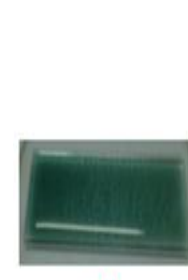

(g)

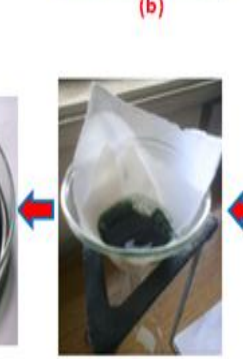

(e)

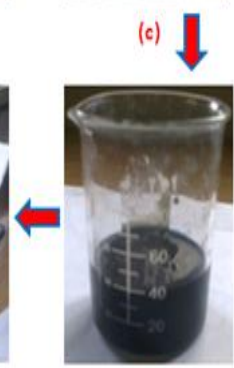

(d)
Fig. 1-Photographic illustration of steps involved in PANI nanofibers synthesis (a) Solutions in beakers contain from left to right aniline hydrochloride and ammonium persulfate, (b) heating up to $60^{\circ} \mathrm{C}$, (c) rapid mixing, (d) PANI undergoing polymerization, (e) filtration and washing, (f) PANI and (g) PANI film on a glass slide

\subsection{Characterization}

Powder X-ray diffraction pattern (XRD) of PANI nanofibers in powdered form was recorded using a (Bruker D-8, Billerica, MA) advance diffractometer with $\mathrm{Cu} \mathrm{Ka}(\lambda=1.5406 \AA)$ as a radiation source, operated at $40 \mathrm{kV}$ and $30 \mathrm{~mA}$ with a scan rate of $0.02^{\circ} / \mathrm{s}$ over the range of $10^{\circ}-80^{\circ}$. XRD analysis was done to identify the crystalline phase, structure and to estimate average crystallite size. The morphological features of PANI nanofibers were studied by using Scanning Electron Microscope (JOEL, FE-SEM 7000). FTIR analysis was performed in the range $4000-400 \mathrm{~cm}^{-1}$ with FTIR spectrophotometer (Perkin Elmer Spectrum BX, Waltham, MA) using PANI nanofibers seeded Potassium Bromide $(\mathrm{KBr})$ powder. UV-VIS spectroscopic measurement of absorbance spectra for PANI nanofibers dispersed in double distilled water was performed using a (Shimatzu 1650PC) UV-VIS spectrophotometer.

\section{RESULTS AND DISCUSSIONS}

Fig. 2 shows the XRD pattern of PANI nanofibers. The XRD pattern reveals crystalline nature of PANI nanofibers. Fig. 2 shows three sharp peaks at $2 \theta=15.14^{\circ}, 19.36^{\circ}, 24.48^{\circ}$, which corresponds to $(011)$, (020) and (200) crystal planes of PANI [4] whereas the peaks corresponding to $2 \theta=44^{\circ}, 48^{\circ}$ and $51.5^{\circ}$ are attributed to the nanocrystalline nature of PANI [5]. The crystalline nature of PANI is due to its nano fibrous form and planer nature of Banzenoid and Quinoid functional groups [6]. The lattice constant (a) was calculated using the formula $a=d \sqrt{h^{2}+k^{2}+l^{2}}$ where $\mathrm{d}$ is interplaner distance and $(h k l)$ are Miller indices. The calculated average value of lattice constant (a) from XRD graph is $a=8.2327 \AA$. The reported values of inter planer distances are $42.14 \mathrm{~nm}$ at $2 \theta=21.10^{\circ}$ and $37.74 \mathrm{~nm}$ at $2 \theta=23.59^{\circ}$ respectively [6]. However the as measured values of inter planer distances from XRD pattern in our experimentation were found to be $45.8 \mathrm{~nm}$ at $2 \theta=19.36^{\circ}$ and $36.3 \mathrm{~nm}$ at $2 \theta=24.48^{\circ}$ respectively. The deviation of the lattice parameters is mainly due to internal stresses which contribute to little shifting of XRD peaks from the expected $2 \theta$ values.

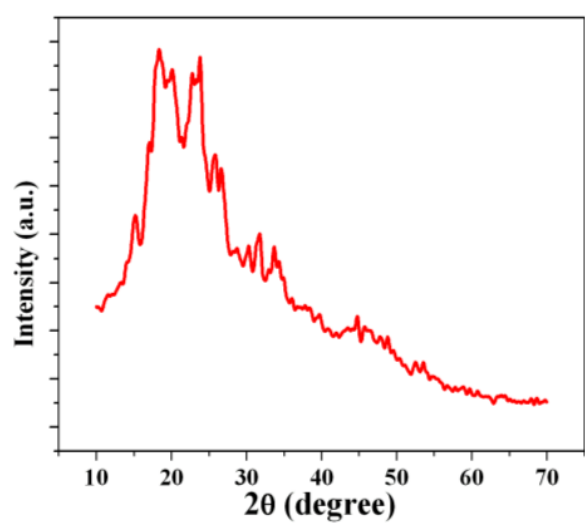

Fig. 2 - XRD pattern of PANI nanofibers

The $2 \theta$ values for prominent peaks, corresponding $(h k l)$ planes and inter-planar distance (d) are represented in Table 1.

Table 1 - XRD analysis of PANI, nanofibers

\begin{tabular}{|c|c|c|}
\hline $2 \theta$ & $(h k l)$ plane & Inter-planar distance $(d)$ in $\AA$ \\
\hline $15.14^{\circ}$ & 011 & 5.85 \\
$19.36^{\circ}$ & 020 & 4.58 \\
$24.48^{\circ}$ & 200 & 3.63 \\
\hline
\end{tabular}

The average crystallite size $(D)$ in $\AA$, was calculated by using Scherer's formula [7] given by (1).

$$
D=\frac{K \lambda}{\beta \cos \theta},
$$

where $K$ is the shape factor usually has a value $0.9, \lambda$ is the X-ray wavelength and $\theta$ the Bragg angle and $\beta$ gives the full width of the half maxima (FWHM). The determined average crystallite size was $30 \mathrm{~nm}$.

The SEM micrograph of as synthesized PANI nanofibers is represented by Fig. 3. The SEM micrograph demonstrates a homogeneous fibrous morphology of PANI nanofibers. It also shows a well formed mesh of interconnected and entangled PANI nano-fibers. The entanglement is mainly attributed to high temperature during actual polymerization process during the synthesis. The interconnected and entangled structure is also due to steady synthesis which allows the easy branching with other fibers growing during polymerization process. This facilitates homogeneous nucleation thereby giving fine uniform and interconnected nanofibers of PANI. On the other hand the low temperature process with stirring and shaking during PANI synthesis encourages heterogeneous nucleation which typically leads to cluster and granule (crystal) formation. The estimated average crystallite size of PANI nanofibers from SEM was $30 \pm 5 \mathrm{~nm}$ which also corroborates the average crystalline size of $30 \mathrm{~nm}$ obtained from XRD. 


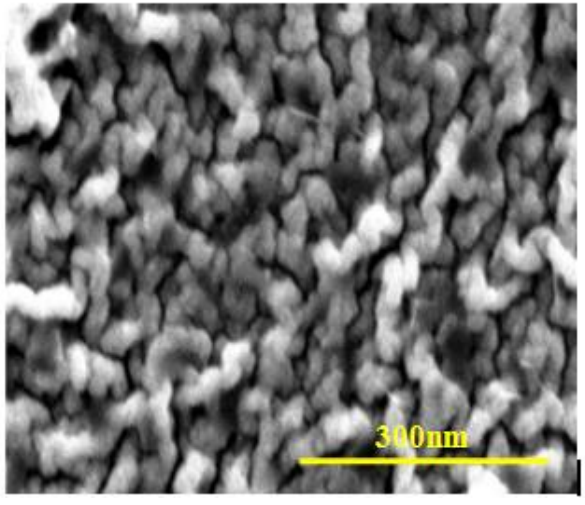

Fig. 3 - SEM micrograph of PANI nanofibers

The FTIR spectrum of PANI is represented in Fig. 4. The Fig. 4 shows vibration band around $3229.24 \mathrm{~cm}^{-1}$ is attributed to the N-H stretching vibrations [8]. The main characteristic peaks at 1568.95, 1475.6, 1297.6, 1139.49 , and $812.94 \mathrm{~cm}^{-1}$. The bands at $1568.95,1475.6$ and 1297.6 are assigned to the stretching mode of $\mathrm{N}=\mathrm{Q}=\mathrm{N}$ ring, $\mathrm{N}-\mathrm{B}-\mathrm{N}$ ring, and $\mathrm{C}-\mathrm{N}\left(\mathrm{C}_{\text {aromatic }}-\mathrm{N}\right)$ deformation, respectively [9] (where $\mathrm{B}$ refers to benzenoid ring and $\mathrm{Q}$ refers to quinonoid ring). Further, the observed band at $812.94 \mathrm{~cm}^{-1}$ attributes to the out-ofplane vibration of $\mathrm{C}-\mathrm{H}$ on 1,4-disubstituted aromatic rings. The presence of characteristic absorption peaks in the FTIR spectrum in Fig. 4 substantiates the formation of PANI nanofibers.

Fig. 5 exhibits UV-VIS absorption spectrum PANI nanofibers. The Fig. 5 shows three major absoption peaks at $256.73,361.17,480.95 \mathrm{~nm}$. The peak observed at $\lambda=256.73 \mathrm{~nm}$ is due to the $\pi-\pi *$ transition of benzenoid ring which is related to the extent of conjugation between the adjacent phenylene rings in the polymeric chain [10]. The less intense band peak at $\lambda=361.17 \mathrm{~nm}$ is due to $\pi$ - $\pi *$ transition while the peak at $480.05 \mathrm{~nm}$ is on account of to polaron- $\pi *$ transition and shift of electron from benzenoid ring to quinonoid ring [11] which represents the protonation of PANI that suggests a conducting state [12]. Further, the peak at $\lambda=820 \mathrm{~nm}$ is due to $\pi$ - polaron transition.

\section{CONCLUSIONS}

PANI nano-fibers have been successfully synthesized by a facile rapid oxidative polymerization of aniline hydro-

\section{REFERENCES}

1. D. Li, J. Huang, R. Kaner, Acc. Chem. Res. 42 No 1, 135 (2009).

2. H. Wei, X. Yan, S. Wu, Z, Luo, S. Wei, Z. Guo. J. Phys. Chem. C 116 No 47, 25052 (2012).

3. Y. Yu, B. Che, Z. Si, L. Li, W. Chen, G. Xue, Synth. Met. 150, 271 (2005).

4. A. Rahy, D. Yang, Mater. Lett. 62 No 28, 4311 (2008).

5. S. Misra, M. Yadav, P. Mathur Grozdanov, Thin Solid Films 514 No 1-2, 272 (2006).

6. B. Kavitha, K. Kumarand, N. Narsimlu, Indian J. Pure Appl. Phys. 55, 207 (2013).

7. H. Klug, L. Alexander, X-Ray Diffraction Procedures: For

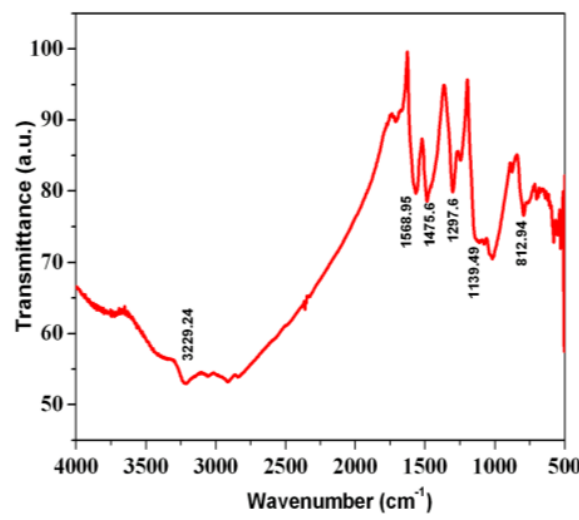

Fig. 4 - FTIR spectrum of PANI nanofibers

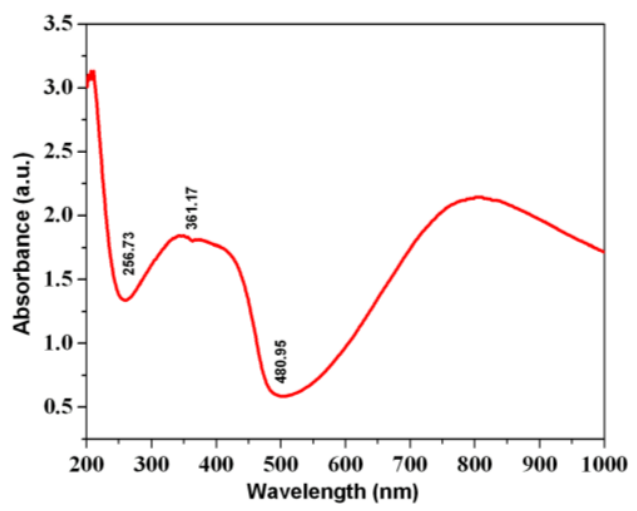

Fig. 5-UV-VIS spectrum on PANI nanofibers.spectrum of PANI nanofibers

chloride and ammonium persulfate at high temperature $\left(60^{\circ} \mathrm{C}\right)$. The XRD study reveals nanocrystalline nature of PANI with average crystallite grain size of $30 \mathrm{~nm}$. SEM studies reveals homogeneous fibrous morphology of PANI nanofibers with a well formed mesh of interconnected and entangled PANI nano-fibers with average diameter of $30 \pm 5 \mathrm{~nm}$ over the scanned area. The presence of characteristic functional groups in FTIR spectrum confirmed formation of PANI.

\section{ACKNOWLEDGEMENTS}

The authors wish to thank for the support extended by the Management, Director, Vishwakarma Institute of Technology and Head, Department of Engineering Sciences and Humanities (DESH), VIT Pune.

Polycrystalline and Amorphous Materials (New York, USA: John Wiley and Sons: 1974).

8. S. Kondawar, S. Acharya, S. Dhakate, Adv. Mater. Lett. 2 No 5, 362 (2011).

9. W. Feng, X. Bai, Y. Lian, J. Liang, X. Wang, K. Yoshino, Carbon 41 No 8, 1551 (2003).

10. X. Jing, Y. Wang, D. Wu, J. Qiang, Ultrasonic. Sonochem. 14 No 1,75 (2007).

11. A. MacDiarmid, A. Epstein, Synth. Met. 65 No 2-3, 103 (1994).

12. R. Tseng, J. Huang, J. Ouyang, R. Kaner, Yang, Nano Lett. 5 No 6, 1077 (2005). 